\title{
Intelligent Transport Systems Exploiting Smart Wireless Sensing Nodes
}

\author{
Filippo G. Praticò (D), Rosario Fedele (D), Sara Pizzi (D), Giuseppe Araniti \\ DIIES Department, University Mediterranea of Reggio Calabria, Reggio Calabria, Italy
}

Received 13 February 2020; accepted 20 April 2020

\begin{abstract}
Future smart cities that will exploit the forthcoming fifth-generation (5G) network will strongly contribute to the development of intelligent transport systems, which will be able to effectively manage changing infrastructural conditions, and to timely exchange crucial information with different stakeholders to improve sustainability and safety. To this end, smart wireless sensing nodes can be effectively exploited. Consequently, the objectives of this study are: 1) to describe the setup and the main potentialities of a wireless sensing system designed for monitoring the environmental and structural conditions on road pavements; 2) to provide an overview about the capability of the 5G network to enable the data exchange required by the designed system. Each sensing node includes different sensors, and is able to send the data gathered from the resource-constrained sensors to a web server used for data processing. Vibrational-, acoustical-, and environmental-related data are used to control traffic pollution, road availability and structural status. The paper describes the in-lab tests carried out on asphalt concrete samples to: i) calibrate the sensors; ii) define structural and environmental thresholds. Results show that the tested node is able to provide reliable data that can be used for the above-described purposes.
\end{abstract}

Keywords: smart cities, smart roads, monitoring system, wireless sensing nodes, environmental and structural conditions, 5G.

\section{Introduction}

The implementation of intelligent transport systems (ITSs) that are able to exploit the forthcoming fifth-generation (5G) network can be a key factor for the success of future smart cities. In more detail, ITSs consisting of smart wireless sensing nodes installed on road infrastructures, which are able to exchange data using the $5 \mathrm{G}$ network, can be used to effectively manage changing environmental and structural conditions, and to timely alert different stakeholders, such as authorities and drivers.

During their service life, road infrastructures are subjected to several actions, such as loads (or, sometimes, overloads), and/or freeze-thaw cycles, etc., which cause annoyances (such as noise, vibration, and air pollution for dwellers near the roads), or permanent distresses (i.e., pavement failures, such as surface and concealed cracks, deformations, etc.) (Moghaddam et al., 2011; Pais et al., 2013; Praticò et al., 2010; Ye et al., 2018). Beside the solutions designed to minimize the effects related to the action mentioned above (such as porous/open graded asphalt concrete (Licitra et al., 2015; Liu et al., 2016), rubberized/poroelastic asphalt concretes (Skov et al., 2014), noise barriers (Ow \& Ghosh, 2017; Potvin et al. 2019), trench barriers (Yao et al., 2019), heavy mass technology (Mhanna et al., 2014), etc.), it is possible to find a multitude of solutions designed to monitor environmental issues (e.g., air pollution (Wong et al., 2019; Zaldei et al., 2017), noise (Praticò et al., 2017), and structural problems (e.g., system based on remote sensing technologies (Schnebele et al., 2015). Among the solutions cited above, wireless sensorbased approaches are becoming more and more popular because of their sustainability (i.e., based on low-power and ultra-low-power devices, fed with renewable energy or self-powered), adaptability (i.e., often based on modular, scalable, and multi-purpose devices), that were proposed in order to monitor traffic noise (Marouf et al., 2018), air pollution (Kaivonen \& Ngai, 2019), stresses, and crack generation and propagation (Alavi et al., 2018).

Data gathered by wireless sensing nodes and required to efficiently monitor both environmental and structural conditions of road pavements must be promptly communicated, especially in critical road conditions, to a web server located in the edge of the network deputed to data processing. Furthermore, the need could rise to deliver from the central controller alert messages or issue commands to sensor nodes in case specific conditions are observed by sensing nodes (e.g., an increase in the periodicity of the transmission of sensed data could be required in case critical conditions are detected). For this reason, a telecommunication infrastructure able to catch the requirements of the intelligent transport system is highly needed. The forthcoming $5 \mathrm{G}$ network answers to this demand by promising data

${ }^{*}$ Corresponding author. E-mail: rosario.fedele@unirc.it

\section{Copyright (C) 2020 The Author(s). Published by VGTU Press}

This is an Open Access article distributed under the terms of the Creative Commons Attribution License (http://creativecommons.org/licenses/by/4.0/), which permits unrestricted use, distribution, and reproduction in any medium, provided the original author and source are credited. 
rates of gigabits per second everywhere, significant reduction of latency, increase of system capacity by $100-1000$ times with respect to the current Long Term Evolution 4th Generation (4G/LTE) standard, and 10-100 times higher density of the connected devices per region (Bajracharya et al., 2018). These significant performance improvement is achieved by means of the following main technologies and approaches: (1) densification of existing cellular networks with the massive addition of small cells and provision of device-to-device (D2D) and machine-to-machine (M2M) communications; (2) multi-antenna transmissions and beamforming; (3) millimeter-wave (mmwave) spectrum; (4) design of a highly flexible and scalable physical layer; (5) simultaneous transmission and reception (e.g., full-duplex (FD) communication); (6) improved energy efficiency by energy-aware communication and energy harvesting; (7) virtualization of wireless resources.

Based on the above, the main objectives of this study are to describe the setup and the main potentialities of a wireless sensing system specifically designed for monitoring road pavements from environmental and structural point of view and to give some guidelines regarding the ability of the $5 \mathrm{G}$ network to deliver the traffic load required by the ITS. Consequently, the paper was organized as follows. Next section (section 1) describes the setup of both the monitoring method, and the wireless sensing system. Subsequently, a description of the tests performed to calibrate a prototype of sensor node is provided (section 2), and the most relevant achieved results are illustrated (section 3). Finally, the main conclusions of the study are drawn.

\section{Method and system setup}

The monitoring method applied in this paper (Fedele et al., 2017, 2018b; Fedele \& Praticò, 2019) uses vibrational and acoustical data to monitor the structural health status (SHS) of road pavements by means of vibro-acoustic signature analyses. In more detail, the method considers the road pavement as a filter. Seismic waves generated by the vehicles, propagate into the road pavement, and are gathered using a properly designed receiver (i.e., an airbornenoise-insulated microphone). Consequently, the above mentioned vibro-acoustic signature can be defined as the acoustic response of the pavement to vehicle loads. As demonstrated in the previous works cited above, meaningful features extracted from the gathered signals can be used to recognize the worsening of the SHS of road pavements due to the occurrence and the propagation of different types of failures (e.g., hidden cracks).

In order to apply this method and make it more sustainable and efficient, a set of wireless sensing nodes can be placed along the roadside. Furthermore, if additional sensors (i.e., temperature and humidity sensor, flame and smoke detector, gas detector, microphone) are integrated into the receiver mentioned above, environmental-related data can be gathered and used to control traffic pollution (i.e., air pollution, and noise), and to detect, in real-time, the occurrence of unusual events on/around the road infrastructure (e.g., a fire or an accident). Finally, all gathered information can be collected and visualized through an ad-hoc designed web platform that end-users can easily access by means of a smartphone or a laptop.

By referring to the wireless sensing system specifically designed for monitoring the structural and environmental conditions of road pavements, each unit of the system (see Figure 1) includes different types of sensors and is able to send the data gathered from the resource-constrained sensors to a web server located in the edge of the network deputed to data processing (using a proper platform dashboard; cf. Figure 1a). Each sensor node (see Figure 1) consists of several sensors (i.e., 3D accelerometer, microphones, temperature and humidity sensor, gas detector, flame and smoke detector), a micro-controller (i.e., Arduino "Leonardo"), a wireless data-transmitter (i.e., Raspberry Pi 3), and a photovoltaic-based stand-alone power-supply system. It is important to underline that, sensing devices should promptly detect critical events while limiting resource consumption (Fedele et al., 2018a). Hence, particular attention was paid to the energy consumption of each part of the system, and to the duty cycles of each sensing node.

The efficiency of the system is based on the sensors and on the power supply system, but strictly relies on the 5G systems, acting as hyper-connected networks mostly consisting of pervasive smart objects. While human communications have been the reference target in previous generations of mobile networks, they will be hugely overtaken by communications among objects in next-generation cellular networks. The 3rd Generation Partnership Project (3GPP) has specified a 5G system architecture aimed to support a wide set of use cases, typically grouped into three classes: 1) enhanced mobile broadband (eMBB); 2) ultra-reliable and low latency communications (URLLC); 3) massive machine-type communications (mMTC) (Akyildiz et al., 2016). Contrary to eMBB that prioritizes peak rates, the focus of mMTC refers to providing scalable connectivity for an increasing number of devices and wide area coverage. A typical example of mMTC is the collection of the measurements from a massive number of sensors. Thus, the application scenario that is the target of this paper perfectly fits the last of the three above mentioned use cases, which requires low power consumption for a huge number of connected devices. In fact, we foresee that a possibly high number of the sensing objects illustrated in the previous subsection will be installed on the roadside with the aim to sense the environment and to periodically transmit gathered information. Standard literature assumption for the mMTC use case are (Bockelmann et al., 2016): (i) small packets, potentially going down to a few bytes; (ii) large number of devices (e.g., up to 300.000 in a single cell); (iii) uplink-dominated transmissions; (iv) low data rates (e.g. around $10 \mathrm{~kb} / \mathrm{s}$ per device); (v) sporadic user activity (e.g., mixed traffic 
models with period and event driven traffic); (vi) low complexity and battery constrained devices. Despite the listed assumptions are more than adequate for the application scenario that is the focus of this work, future work will be tailored to a more accurate evaluation of the capability of the $5 \mathrm{G}$ network to enable the data exchange required for the operation of the designed wireless sensing system.

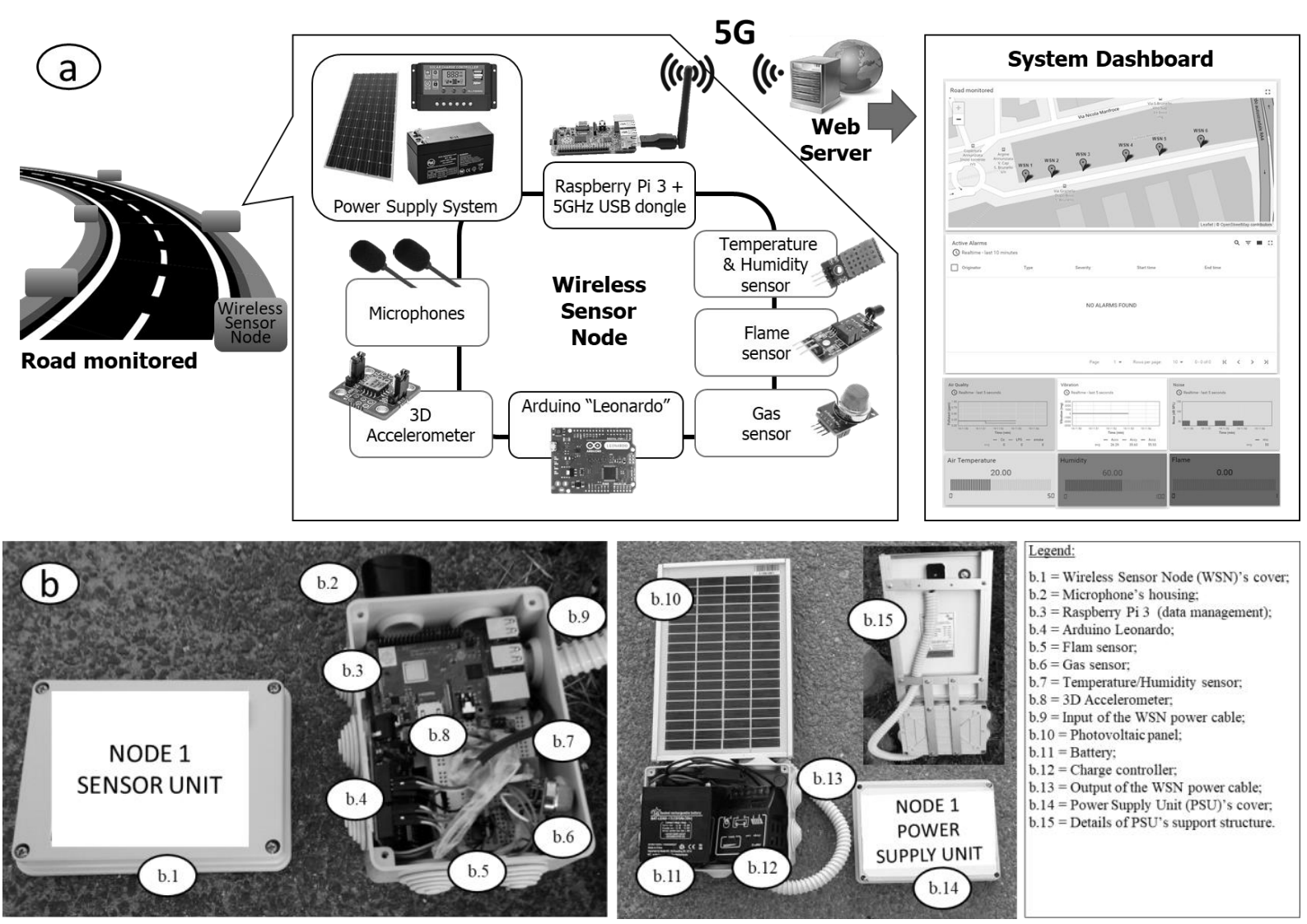

Figure 1. Wireless sensor node used in this study: a) schematic representation; b) picture of the first prototype

\section{Sensor node calibration}

This section presents the results of the tests that were carried out in laboratory using a first prototype of the sensing node (see Figure 2).

With the aim to replicate on-site working conditions, the node was glued on an asphalt concrete slab. Subsequently, the slab was properly loaded to calibrate the 3D MEMS accelerometer (ADXL355; inside the box in Figure 1b) and the microphone (Knowles FB-EM-30343-000; inside the box in Figure 1b) used for the derivation of the vibro-acoustic signature. Hence, two relative calibrations were carried out. In more detail, the accelerometer was calibrated using a reference measurement chain that consisted of a 3D accelerometer (Brüel \& Kjær 4529B; glued on the slab in Figure 1b), an analog-to-digital board (Brüel \& Kjær LAN-XI 3160), and a laptop where the software Brüel \& Kjær BK Connect is installed. At the same time, a sound level meter (Norsonic Nor140; cf. Figure 1b) was used to calibrate one of the microphones of the sensing node (i.e., the microphone deputed to the detection of the vibro-acoustic signature). Signals as similar as possible to a Dirac impulse (which would allow obtaining flat spectra with a bandwidth as long as possible; cf. Seimens, 2019) were generated using an impact hammer (Brüel \& Kjær 8206-002, equipped with a metallic tip) hitting a metallic platelet glued on the slab surface. The measurements were repeated several times, hitting the slab 5 times per measurement. Seismic and acoustic responses of the slab were recorded using the sensors of the sensing node and the reference devices, using as sampling frequencies 1) $36 \mathrm{kHz}$ and $40 \mathrm{kHz}$ for microphone and sound level meter, respectively. 2) $1.9 \mathrm{kHz}$ and $653 \mathrm{kHz}$ for the node-, and reference-related accelerometer, respectively. Subsequently, the 1/3 octave band spectra were obtained and compared to derive two calibration vectors. Furthermore, fire and smoke (see Figure 3b, and c) were generated in a controlled environment to test the response of the related sensors, while a lighter was used to test the response of the gas sensor. 

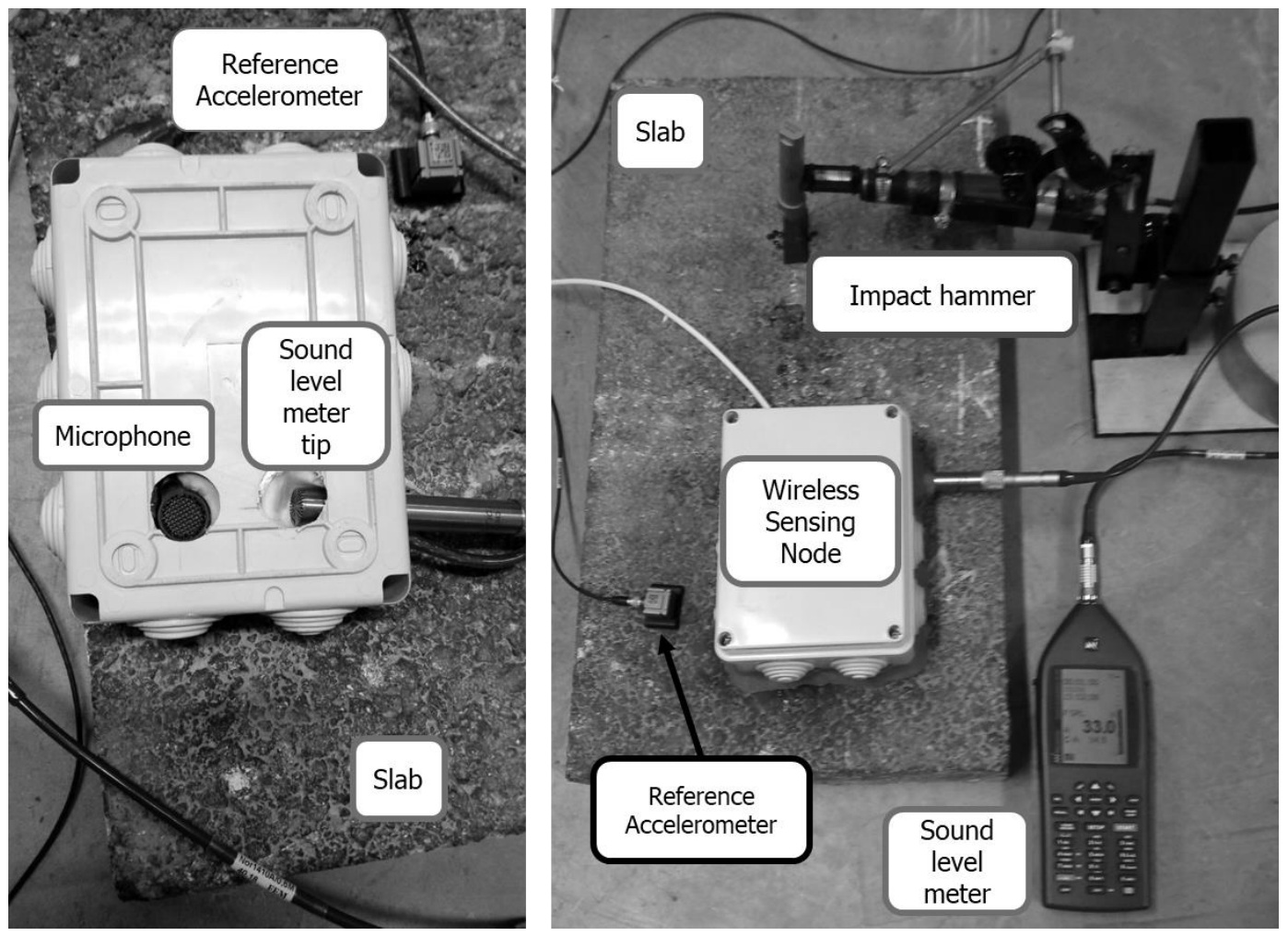

Figure 2. Wireless sensor node during the in-lab tests: calibration of 3D accelerometer and microphone
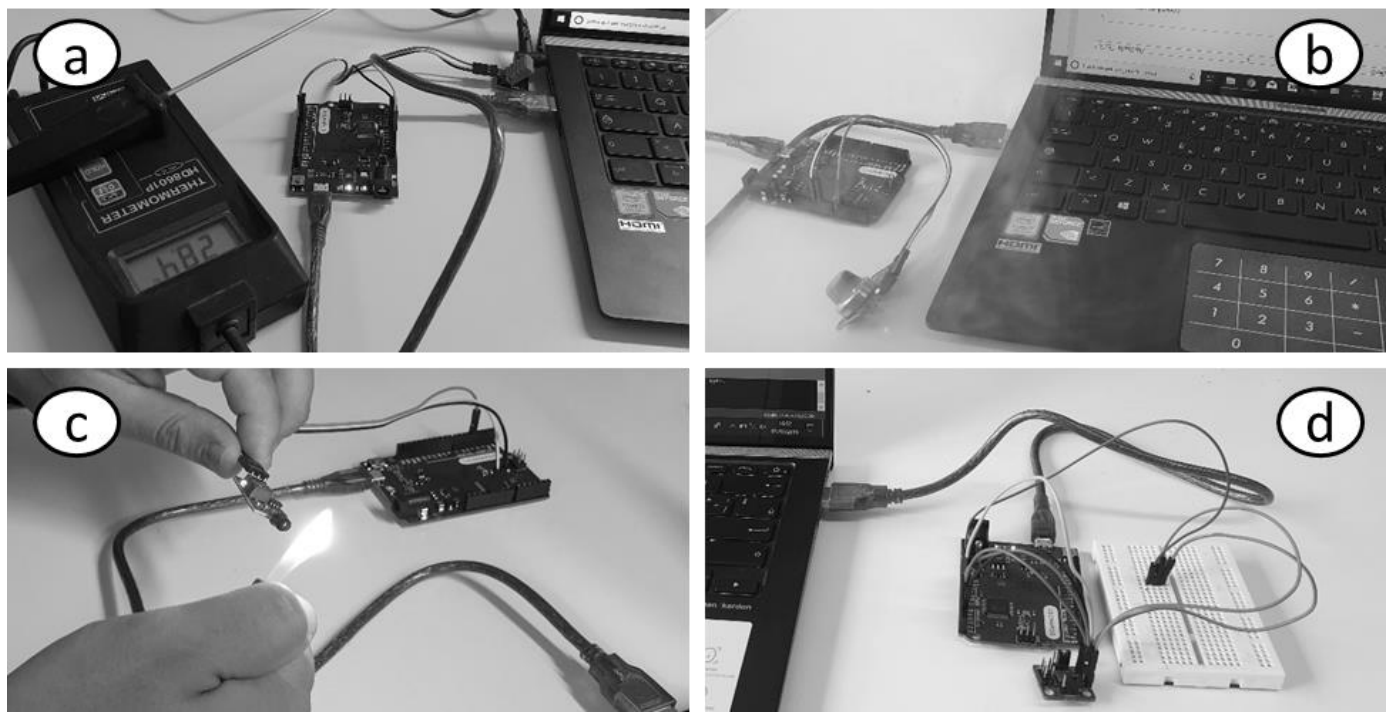

Figure 3. Wireless sensing node during the in-lab tests: definition of threshold for a) thermometer; b) smoke; c) flame, and d) acceleration

\section{Results and discussions}

The following figures show seismic and acoustic signals gathered as explained previously, and the related spectra derived using a proper Matlab code. In particular, Figure 4 shows one of the impulse load generated by the impact hammer and the related spectrum. Figure 5 shows one of the seismic responses of the slab (acceleration along 3 axes versus frequency) measured by the $3 \mathrm{D}$ accelerometer of the node in the time (Figure 5a), and in the frequency (Figure 5b) domains, while Figure 5c, and Figure 5d report the same response from the reference accelerometer. 

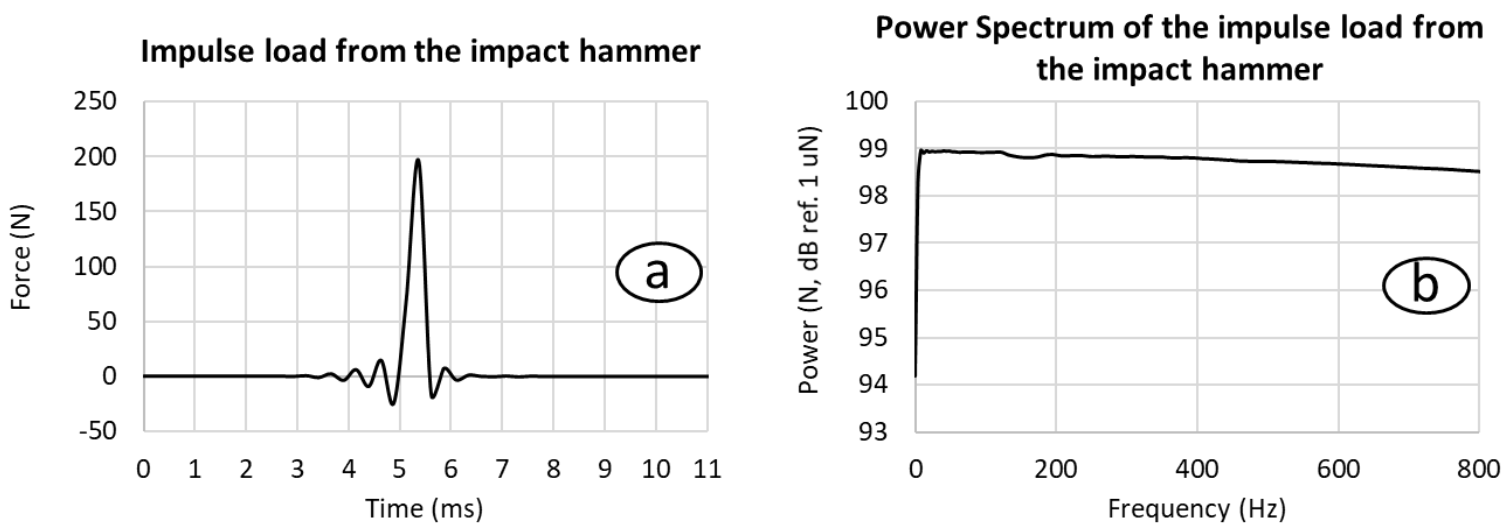

Figure 4. Example of (a) impulse load generated by the impact hammer, and (b) the related spectrum
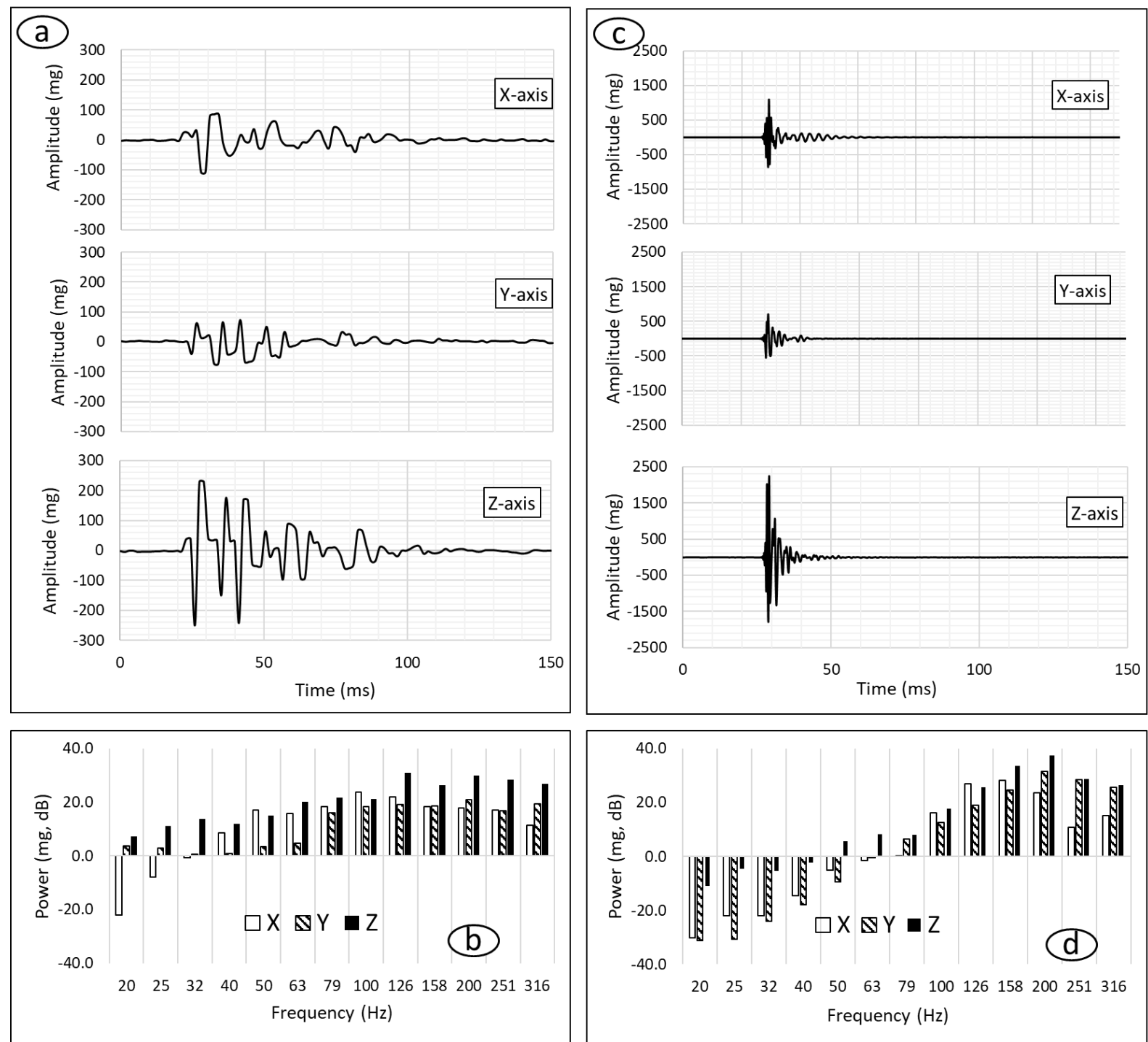

Figure 5. Example of seismic responses of the slab to a given impulse load: a) signal detected by the accelerometer to be calibrated; b) spectrum of the signal (a); c) signal detected by the reference accelerometer; d) spectrum of the signal (c)

Figure 6 refers to the measurements carried out to calibrate the node's microphone. In particular, Figure $6 \mathrm{a}$ shows one of the acoustic signals gathered using the microphone to calibrate, while Figure $6 \mathrm{~b}$ shows the related spectrum with and without the A-weighting (i.e., a standard weighting of the audible frequencies, $20 \mathrm{~Hz} \div 20 \mathrm{kHz}$, designed to consider the human ear's response to noise, where the attenuation at $1 \mathrm{kHz}$ is 0 and nonzero otherwise; 
while Z-weighting does not attenuate the spectrum, i.e. is $0 \mathrm{~dB}$ in all the frequency bands). Figure $6 \mathrm{c}$ shows the acoustic responses (frequency domain) detected by the sound level meter, with (A-weighted spectrum) and without (Z-weighted spectrum) weighting automatically applied by the device. Results in Figure 6 show that the sound level meter provides results that are, as expected, more precise and detailed than those obtained by the microphone of the sensing node. Despite this, a proper calibration vector can be derived by subtracting the power of each $1 / 3$ octave band of the spectra in Figure 6b, and c.
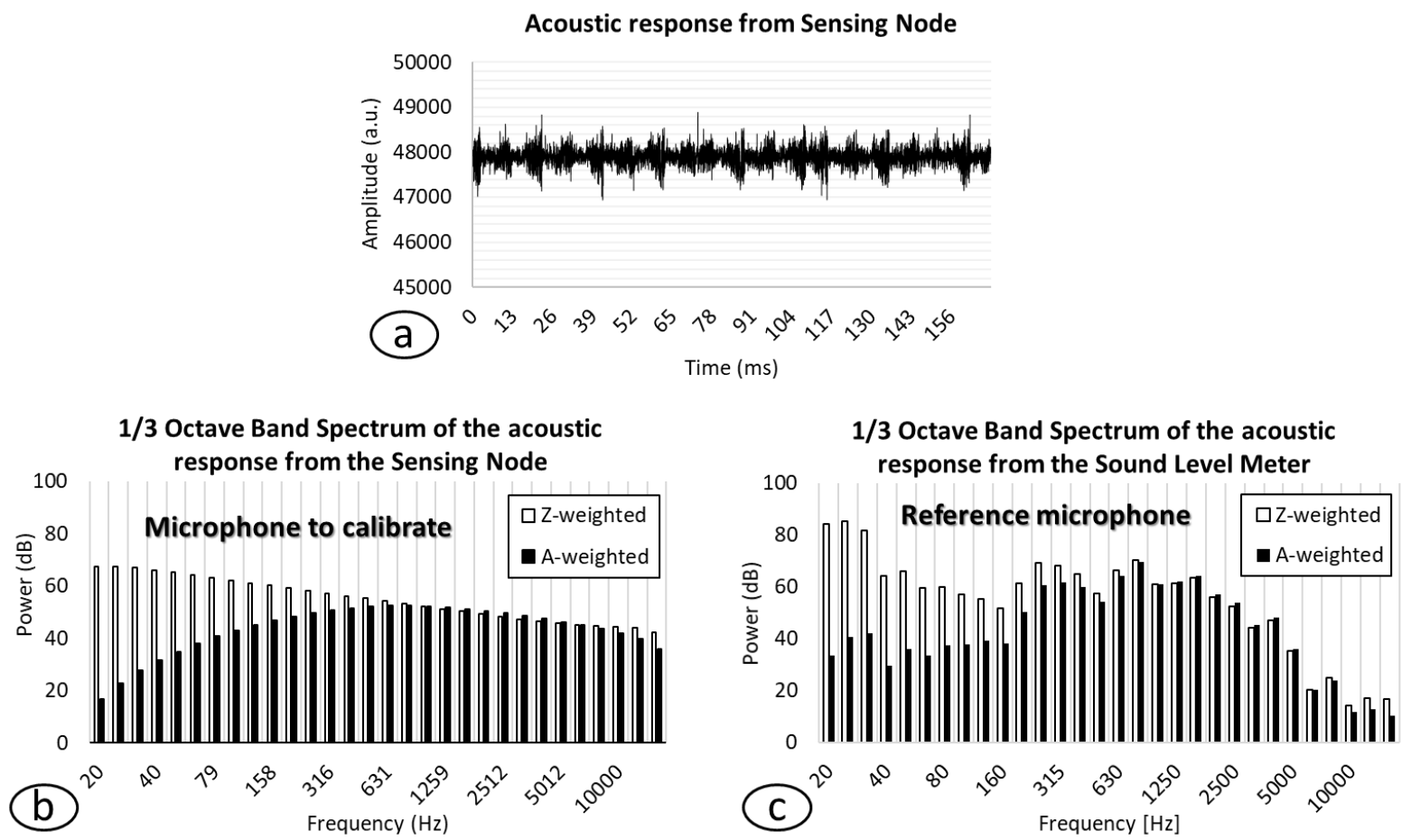

Figure 6. Example of acoustic responses of the slab to a given impulse load: a) signal detected by the microphone to be calibrated; b) spectrum of the signal (a); c) spectrum of the signal detected by the sound level meter

As described above, the monitoring method aims at detecting the vibro-acoustic signature of the monitored object (e.g., road pavement) using an air-borne noise insulated microphone. For this reason, a proper insulation of the microphone inside the sensing node (i.e., box in Figure 1b) is needed. Consequently, the effect of the node case, and the insulating material (that was insert on the node case) was studied using the sound level meter to detect the Sound Pressure Level (SPL). In particular, the background noise was measured while the microphone of the sound level meter was inside and outside the sensing node case. Figure 7 shows the region of the spectrum that was affected by the insulation $(100 \div 5000 \mathrm{~Hz})$, and based on the results provided by the sound level meter, the average Leq undergoes a reduction of $36.2 \mathrm{dBA}$.

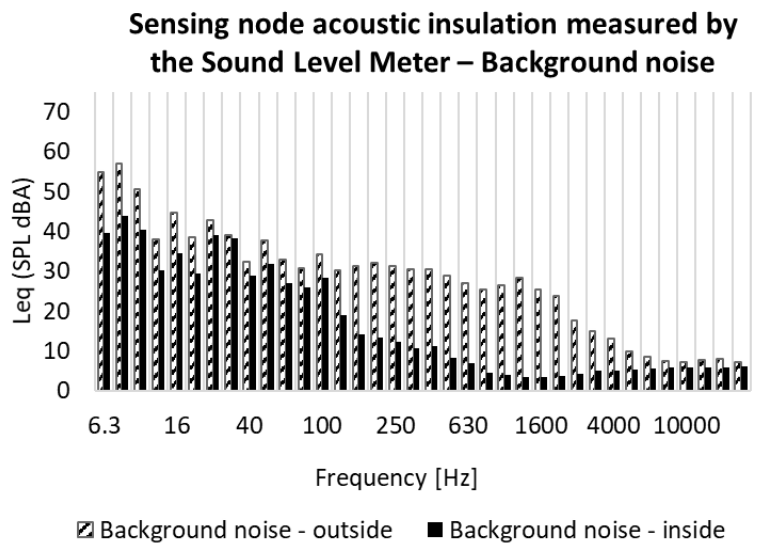

Figure 7. Microphone insolation due to the isolating material put on the node case: a) background noise; b) during 5 hits of the impact hammer 
Results show that the node is able to provide reliable data, which can be used to recognize the variation of the road conditions, and to trigger alerts/alarms when the traffic flow is interrupted and the structural and environmental health are compromised.

Finally, a dashboard (Figure 1a) was created to allow the users of the proposed system to visualize sensor data, statistics, and presence/absence of alerts/alarms in real-time. Based on the test showed in Figure 2 and 3 , the following thresholds were defined to trigger the three levels (Minor, Major, Critical) of alarm: 1) Temperature: medium-high $=30{ }^{\circ} \mathrm{C} \div 40{ }^{\circ} \mathrm{C}$, high $=40{ }^{\circ} \mathrm{C} \div 50{ }^{\circ} \mathrm{C}$, critical $>50{ }^{\circ} \mathrm{C}$; 2) Humidity: medium-high $=70 \% \div 80 \%$, high $=80 \% \div 90 \%$, critical $>90 \%$; 3) Carbon Monoxide (CO; parts-per-million, ppm): medium-high $=0 \mathrm{ppm} \div$ $5 \mathrm{ppm}$, high $=25 \mathrm{ppm} \div 35 \mathrm{ppm}$, critical $>35 \mathrm{ppm}$; 4) Liquefied Propane Gas (LPG): medium-high $=0 \mathrm{ppm} \div$ $5000 \mathrm{ppm}$, high $=5000 \mathrm{ppm} \div 10000 \mathrm{ppm}$, critical $>10000 \mathrm{ppm}$; 5) Flame: no flame $=0$, flame detected = 1; 6) Smoke thresholds are the same of the ones above for LPG; 7) Absolute values of the Acceleration (1 g $\approx$ $9.81 \mathrm{~m} / \mathrm{s}^{2}$ ): medium-high $=1.5 \mathrm{~g} \div 2 \mathrm{~g}$, high $=2 \mathrm{~g} \div 3 \mathrm{~g}$, critical $>3 \mathrm{~g} ; 8$ ) Noise (Sound Pressure Level, SPL, dB): medium-high $=50 \mathrm{~dB} \div 60 \mathrm{~dB}$, high $60 \mathrm{~dB} \div 70 \mathrm{~dB}$, critical $>70 \mathrm{~dB}$.

\section{Conclusions}

The sustainability and safety of intelligent transport systems, which will be part of the future smart cities, should be designed to effectively manage environmental and structural changing conditions, and to timely exchange crucial information with different stakeholders (e.g., road agency, authorities, drivers). To this end, wireless sensing nodes able to timely communicate sensed data over the fifth-generation (5G) network can be exploited. The main potentialities of a wireless sensing system designed for road pavement environmental and structural monitoring, and an overview about the capability of the $5 \mathrm{G}$ network to enable the data exchange required by the designed system have been studied. In more detail, the sensors of a node prototype were tested in laboratory conditions aiming at (1) calibrating the microphone and the $3 \mathrm{D}$ accelerometer used for the environmental/structural monitoring, and (2) defining proper thresholds for environmental-, and structural-related parameters. Results show that the node is able to provide reliable data, which can be used to recognize the variation of the road conditions, and to trigger alerts/alarms when the traffic flow is interrupted and the structural and environmental health are compromised. Future work will be tailored to a more accurate evaluation of 1) The performances of the wireless sensing system in operational conditions (i.e., sensing node installed roadside). 2) The capability of the 5G network to enable a proper (safe, fast, and less-consuming) data exchange required by the designed wireless sensing system.

\section{Funding}

This work was supported by the ongoing Italian project "USR342" (i.e., urban safety, sustainability, and resilience: 3 paving solutions, 4 sets of modules, 2 platforms; reference number: PRIN2017XYM8KC).

\section{Author contributions}

All the authors conceived the study. Praticò and Fedele were responsible for data collection and analysis. Pizzi and Araniti were responsible for data transmission and results interpretation.

\section{Disclosure statement}

Authors formally declare that they have not competing financial, professional, or personal interests from other parties.

\section{References}

Akyildiz, I. F., Nie, S., Lin, S. C., \& Chandrasekaran, M. (2016). 5G roadmap: 10 key enabling technologies. Computer Networks. 106, 17-48. https://doi.org/10.1016/j.comnet.2016.06.010

Alavi, A. H., Jiao, P., Buttlar, W. G., \& Lajnef, N. (2018). Internet of Things-enabled smart cities: State-of-the-art and future trends. Measurement: Journal of the International Measurement Confederation, 129, 589-606. https://doi.org/10.1016/j.measurement.2018.07.067

Bajracharya, R., Shrestha, R., Ali, R., Musaddiq, A., \& Kim, S. W. (2018). LWA in 5G: State-of-the-art architecture, opportunities, and research challenges. IEEE Communications Magazine, 56(10), 134-141. https://doi.org/10.1109/MCOM.2018.1701177

Bockelmann, C., Pratas, N., Nikopour, H., Au, K., Svensson, T., Stefanovic, C., Popovski, P., \& Dekorsy, A. (2016). Massive machine-type communications in 5g: Physical and MAC-layer solutions. IEEE Communications Magazine, 54(9), 59-65. https://doi.org/10.1109/MCOM.2016.7565189

Fedele, R., Merenda, M., Praticò, F. G., Carotenuto, R., \& Della Corte, F. G. (2018a). Energy harvesting for IoT road monitoring systems. Instrumentation Mesure Metrologie, 17(4), 605-623. https://doi.org/10.3166/i2m.17.605-623 
Fedele, R., \& Praticò, F. G. (2019). Monitoring infrastructure asset through its acoustic signature. INTER-NOISE and NOISECON Congress and Conference Proceedings, 259(8), 1970-1981.

Fedele, R., Praticò, F. G., Carotenuto, R., \& Della Corte, F. G. (2017). Structural health monitoring of pavement assets through acoustic signature. In $10^{\text {th }}$ International Conference on the Bearing Capacity of Roads, Railways and Airfields (BCRRA 2017). https://doi.org/10.1201/9781315100333-127

Fedele, R., Praticò, F. G., Carotenuto, R., \& Della Corte, F. G. (2018b). Energy savings in transportation: setting up an innovative SHM method. Mathematical Modelling of Engineering Problems, 5(4), 323-330. https://doi.org/10.18280/mmep.050408

Kaivonen, S., \& Ngai, E. C. H. (2019). Real-time air pollution monitoring with sensors on city bus. Digital Communications and Networks, 6(1), 23-30. https://doi.org/10.1016/j.dcan.2019.03.003

Licitra, G., Cerchiai, M., Teti, L., Ascari, E., Bianco, F., \& Chetoni, M. (2015). Performance assessment of low-noise road surfaces in the leopoldo project: Comparison and validation of different measurement methods. Coatings, 5(1), 3-25. https://doi.org/10.3390/coatings5010003

Liu, M., Huang, X., \& Xue, G. (2016). Effects of double layer porous asphalt pavement of urban streets on noise reduction. International Journal of Sustainable Built Environment, 5(1), 183-196. https://doi.org/10.1016/j.ijsbe.2016.02.001

Marouf, S., Bell, M. C., Goodman, P., Namdeo, A., \& Neasham, J. (2018). Pervasive wireless sensors: A new monitoring tool for road traffic noise evaluation. Applied Acoustics, 135, 48-59. https://doi.org/10.1016/j.apacoust.2018.01.017

Mhanna, M., Shahrour, I., Sadek, M., \& Dunez, P. (2014). Efficiency of heavy mass technology in traffic vibration reduction: Experimental and numerical investigation. Computers and Geotechnics, 55, 141-149. https://doi.org/10.1016/j.compgeo.2013.08.002

Moghaddam, T. B., Karim, M. R., \& Abdelaziz, M. (2011). A review on fatigue and rutting performance of asphalt mixes. Scientific Research and Essays, 6(4), 670-682. http://www.academicjournals.org/SRE

Ow, L. F., \& Ghosh, S. (2017). Technical note Urban cities and road traffic noise: Reduction through vegetation. Applied Acoustics, 120, 15-20. https://doi.org/10.1016/j.apacoust.2017.01.007

Pais, J. C., Amorim, S. I. R., \& Minhoto, M. J. C. (2013). Impact of traffic overload on road pavement performance. Journal of Transportation Engineering, 139(9). https://doi.org/10.1061/(ASCE)TE.1943-5436.0000571

Potvin, S., Apparicio, P., \& Séguin, A. M. (2019). The spatial distribution of noise barriers in Montreal: A barrier to achieve environmental equity. Transportation Research Part D: Transport and Environment, 72, 83-97. https://doi.org/10.1016/j.trd.2019.04.011

Praticò, F. G., Fedele, R., \& Vizzari, D. (2017). Significance and reliability of absorption spectra of quiet pavements. Construction and Building Materials, 140, 274-281. https://doi.org/10.1016/j.conbuildmat.2017.02.130

Praticò, F. G., Ammendola, R., \& Moro, A. (2010). Factors affecting the environmental impact of pavement wear. Transportation Research Part D: Transport and Environment, 15(3), 127-133. https://doi.org/10.1016/j.trd.2009.12.002

Schnebele, E., Tanyu, B. F., Cervone, G., \& Waters, N. (2015). Review of remote sensing methodologies for pavement management and assessment. European Transport Research Review, 7, 7. https://doi.org/10.1007/s12544-015-0156-6

Skov, R. S. H., Andersen, B., Bendtsen, H., \& Cesbron, J. (2014). Laboratory measurements on noise reducing PERS test slabs. Proceedings of Forum Acusticum, 7-12 September, 2014. Krakow, Poland.

Wong, P. P. Y., Lai, P. C., Allen, R., Cheng, W., Lee, M., Tsui, A., Tang, R., Thach, T. Q., Tian, L., Brauer, M., \& Barratt, B. (2019). Vertical monitoring of traffic-related air pollution (TRAP) in urban street canyons of Hong Kong. Science of the Total Environment, 670, 696-703. https://doi.org/10.1016/j.scitotenv.2019.03.224

Yao, J., Zhao, R., Zhang, N., \& Yang, D. (2019). Vibration isolation effect study of in-filled trench barriers to train-induced environmental vibrations. Soil Dynamics and Earthquake Engineering, 125, 105741. https://doi.org/10.1016/j.soildyn.2019.105741

Ye, Z., Lu, Y., \& Wang, L. (2018). Investigating the pavement vibration response for roadway service condition evaluation. Advances in Civil Engineering, 2018, ID 2714657. https://doi.org/10.1155/2018/2714657

Zaldei, A., Camilli, F., De Filippis, T., Di Gennaro, F., Di Lonardo, S., Dini, F., Gioli, B., Gualtieri, G., Matese, A., Nunziati, W., Rocchi, L., Toscano, P., \& Vagnoli, C. (2017). An integrated low-cost road traffic and air pollution monitoring platform for next citizen observatories. Transportation Research Procedia, 24, 531-538. https://doi.org/10.1016/j.trpro.2017.06.002 